\title{
Study on Impeller Fracture Model Based on Vibration Characteristics and Fractal Theory
}

\author{
Xiaolong Zhang, Ruishan Yuan, and Yonghui Xie \\ School of Energy and Power Engineering, Xi'an Jiaotong University, Xi'an, Shaanxi 710049, China \\ Correspondence should be addressed to Yonghui Xie; yhxie@mail.xjtu.edu.cn
}

Received 16 June 2014; Accepted 14 August 2014; Published 12 October 2014

Academic Editor: Xiao-Jun Yang

Copyright ( 2015 Xiaolong Zhang et al. This is an open access article distributed under the Creative Commons Attribution License, which permits unrestricted use, distribution, and reproduction in any medium, provided the original work is properly cited.

\begin{abstract}
During the operation of centrifugal compressor, failure easily occurs in the presence of complicated external forces. The failure process characterizes with strong nonlinearity, and hence it is difficult to be described by conventional methods. In this paper, firstly, the cracks in different positions are described using crack fractal theory. The basic failure modes of the impeller are summarized. Secondly, a three-dimensional finite element model of the impeller is constructed. Then the von Mises stress under the centrifugal force is calculated, and the corresponding impeller failure process is simulated by "element life and death technology" in ANSYS. Finally, the impeller failure mechanism is analyzed. It can be found that the static stress is not the main cause of the impeller failure, and the dynamic characteristics of the impeller are not perfect because of the pitch vibration modes which appeared in the investigated frequency range. Meanwhile, the natural frequency of the impeller also cannot avoid the frequency of the excitation force.
\end{abstract}

\section{Introduction}

Centrifugal compressors are widely used in various fields such as aviation, metallurgy, petrifaction, environmental protection, and pharmacy industry. Centrifugal impeller is a core component of centrifugal compressors, which is composed of hub, blades, and shrouds [1]. With the development of processing technology, welding impeller has been extensively used. Particularly, more and more high-speed centrifugal impellers in recent years are welded. The failure of centrifugal impeller with high-speed rotation is very serious. It not only affects the normal function of machinery, but also threats the safety of operator.

Scholars at home and aboard have carried out significative research on the impeller failure modes, summing up the following two main reasons for the cause of the impeller failure fracture: one is its own defects or material processing defects and the other is the alternating stress fatigue. The material and manufacturing defects are well understood [28]. It is shown that alternating stress in internal compressor is mainly from unsteady flow and aerodynamic loads [5, 912]. Combining the preceding research, the fatigue life design theory is proposed $[3,4,13,14]$.
Fracture mechanics is developed in the recent decades. Based on the macroscopic continuum mechanics, fracture mechanics can be applied to describe propagation and unstability of crack.

Fractal theory can accurately describe the crack process. Since 1975, Benoit B. Mandelbrot put forward fractal theory; the fractal theory has been widely applied. Ritchie and Olff [15] studied the relationship between the space scaling and biodiversity, providing a model to calculate the species diversity by using habitat fractal dimension. Lizzi et al. [16] studied the emergency management on multiband fractal antenna wireless communication systems. Taylor et al. [17] researched Pollock watercolor by using fractal theory. Mirny [18] used the fractal theory to describe chromosome. The fractal structures are also widely observed in dynamical systems $[19,20]$ and can induce long irregular transients [21].

In order to accurately reveal the failure mechanisms of centrifugal compressor impeller, we use two basic fundamental concepts of fractal theory, that is, fractal dimension and fractal length, and to describe the impeller fracture cracks, based on the sufficient analysis of centrifugal compressor impeller failure modes, the centrifugal compressor impeller common failure modes are summarized. Combining with 
the actual situation, the three-dimensional finite element model of a centrifugal compressor impeller is constructed, and the failure mechanisms are simulated.

\section{Impeller Failure Mode Analysis}

2.1. Description on Impeller Cracks. Impeller suffered from a variety of force including centrifugal force, air force, and other exciting force which lead to failure. The process has strong nonlinearity, which is mainly caused by two reasons. First, the external load of impeller is nonlinear; second, impeller failure process is nonlinear, which is difficult to be described by the conventional methods. Since in the failure process, a variety of different scales of fracture, which is distributed around the failure surface, displays a strong similarity. Therefore, to use fractal theory to describe the failure process is an effective method.

The specific process can be described by two basic concepts, fractal dimension and fractal length. Assuming an impeller cracks into $N$ pars and one part is similar to another one with similarity ratio $\beta$, then the similar dimension can be expressed as

$$
D=\frac{\ln N}{\ln (1 / \beta)}=-\frac{\ln N}{\ln \beta}
$$

and similar dimension $D$ does not need to be integers.

Mandelbrot gives the estimation model to calculate length of the fractal curve

$$
L=L_{0} \varepsilon^{1-D},
$$

where $L$ is fractal curves' Euclidean length, $L_{0}$ is the initial operation length of the fractal curve, $\varepsilon$ is fractal curve' scaling, and $D$ is the fractal dimension.

Based on the above fractal theory, with specific forms of centrifugal compressor, it is easy to present the impeller failure modes.

2.2. Centrifugal Compressor Impeller Failures Modes. Figure 1 is the sectional view for a centrifugal compressor. The rotor is constituted by shaft and three centrifugal impellers (shown in Figure 2). Centrifugal impeller is welded together by three bodies, that is, hub, blades, and shroud, which are made of carbon quenched and tempered steel. By a period of operation time, this centrifugal impeller failure occurs frequently (as shown in Figures 3(a), 3(b), and 3(c)). In some serious spots, the impeller could be broken into pieces (shown in Figure 4). According to the collected data from the scene, its life ranges from a few days to more than a year. Figuring out the mechanism and finally solving this problem are extremely important.

There are three main failure modes based on the failure characteristics of the scene macrocracks.

Failure Mode 1. Macrocracks are distributed in the vicinity of blades and shroud welding arc zone (hereinafter referred to

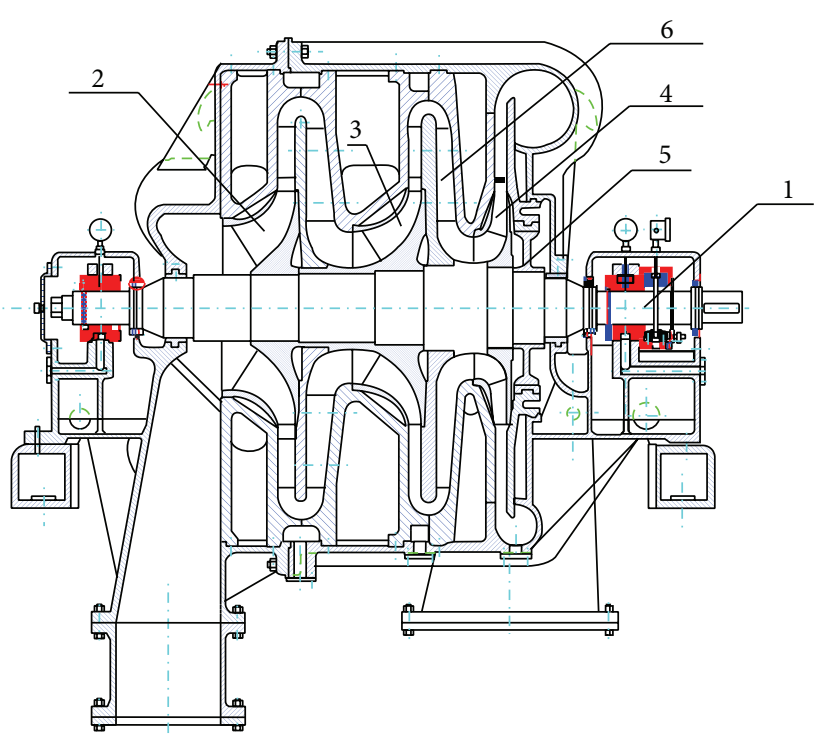

FIGURE 1: Sectional view of a compressor.

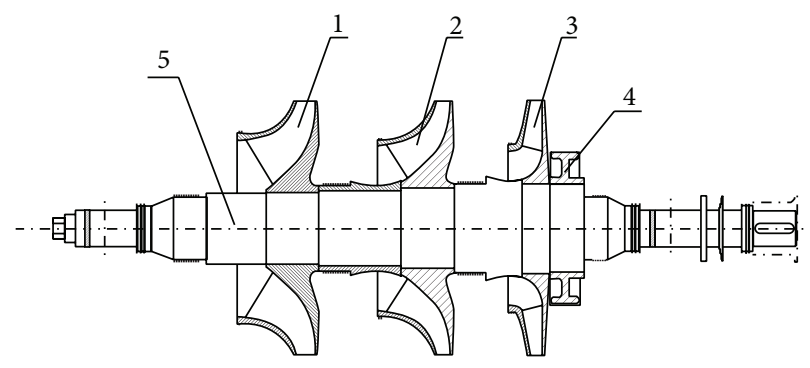

Figure 2: The rotor

as point $\mathrm{A}$ ), and the shroud radially cracks in the blade inlet side (shown in Figure 5(a)).

Failure Mode 2. Macrocracks are distributed in the vicinity of blades and hub welding arc zone (hereinafter referred to as point B) and break circumferentially (shown in Figure 5(b)).

Failure Mode 3. Macrocracks are distributed in the vicinity of blades and hub welding arc zone (hereinafter referred to as point $\mathrm{C}$ ) and crack outwardly from the blade along the direction of the weld (shown in Figure 5(c)).

By comparing the three failure modes mentioned above, the cracks show the feature of self-similarity and can be described by fractal theory, although the cracks locations are different. Fractal dimension and fractal length can be calculated using formulas (1) and (2).

\section{Numerical Simulations of Impeller Failure Modes}

3.1. Elastic Strain Energy Density Unit. According to [22-27], the research focuses on the deformation of a unit volume element under external force. Assuming that there is no heat exchange with the outside, that is, a closed system, the total energy generated from the external power is $W$. According 


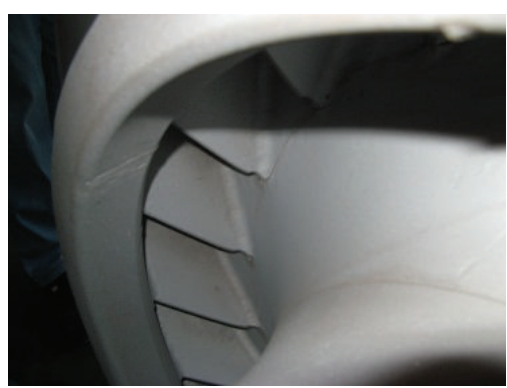

(a) The eyes of the shroud weld

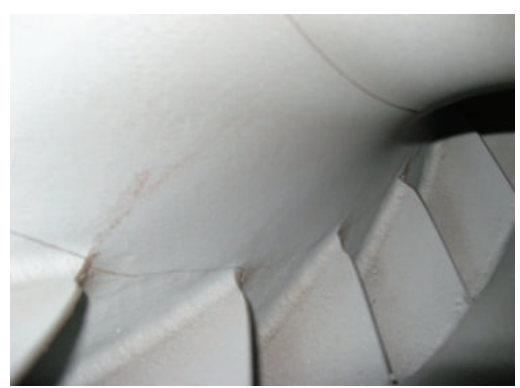

(b) Hub weld

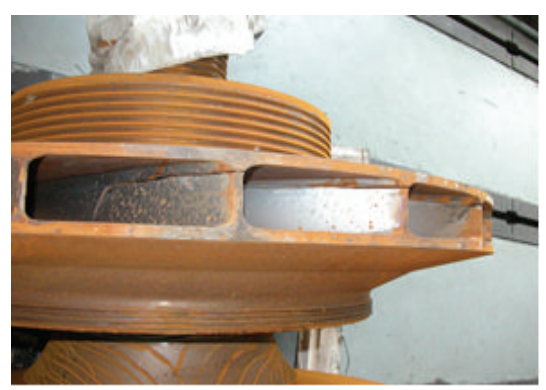

(c) Weld between the hub and the blade

FIgURE 3: Impeller failures.

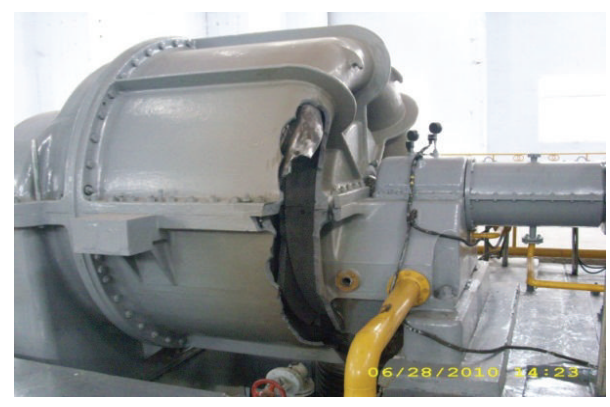

Figure 4: Typical failure impeller pieces.

to the first law of thermodynamics, the total strain energy $W$ generated in the unit is

$$
W=W^{d}+W^{e}
$$

where $W^{d}$ is strain energy dissipation and $W^{e}$ is releasable elastic strain energy of a unit.

Figure 6 shows the value of the element energy dissipation and releasable strain energy per unit volume. The area of $W_{i}^{d}$ indicates the energy consumed when the unit damage and the plastic deformation occur. The shaded area $W_{i}^{e}$ represents the releasable strain energy stored in elements, and $E_{i}$ is unloaded elastic modulus. From the thermodynamic view, the energy dissipation is unidirectional and irreversible, but the energy release is bidirectional, as long as certain conditions are satisfied.

We then define the amount of energy damage of per unit volume:

$$
\omega=\frac{W^{d}}{W^{c}},
$$

where $W^{c}$ is material critical strain dissipation energy and it is a constant which is irrelevant to the state of stress and is determined by the material test.

Under any stress state, $0 \leq \omega \leq 1$, when $\omega=1$, the material loses strength; that is,

$$
W_{\max }^{d}=W^{c}
$$

Strain energy density $\rho$ is defined as $d W / d V$, and because the above theoretical formulas are derived based on unit volume, (3), (4), and (5) evolve as follows:

$$
\begin{gathered}
\rho=\rho^{d}+\rho^{e}, \\
\omega=\frac{\rho^{d}}{\rho^{c}} \\
\rho_{\max }^{d}=\rho^{c},
\end{gathered}
$$

where $\rho^{d}$ is the strain energy dissipation density of a unit, $\rho^{e}$ is releasable elastic strain energy density of a unit, and $\rho^{c}$ is the critical strain energy density dissipated when a unit loses strength.

According to (6) we can obtain

$$
\rho=\rho^{d}+\rho^{e}=\omega \rho^{c}+\rho^{e} .
$$

Alloy material failure usually goes through several stages as shown in Figure 7: elastic stage, yield stage, strength hardening stage, and local plastic deformation stage. In other words, before the material enters the yield stage, $\rho^{e}$ reaches the maximum value $\rho_{\max }^{e} \cdot \rho_{\max }^{e}$ can be obtained based on material test and the material loses strength when $\omega=1$; so formula (7) becomes

$$
\rho_{\max }=\rho_{\max }^{d}+\rho_{\max }^{e}=\rho^{c}+\rho_{\max }^{e} .
$$

Equation (8) is the criteria of unit failure (strength loss). That is, the unit failure occurs when the total unit strain energy density reaches the maximum value for the sum of material critical strain energy dissipation density and releasable elastic strain energy density of a unit.

3.2. Finite Element Model of the Impeller. The threedimensional finite element model of the impeller is built according to the design drawings, with a total of 19 blades, uniformly distributed in the circumferential direction, and can be described as cycle symmetry structure. In order to reduce the computation cost, symmetric cycle model is used. We can focus on 1/19 of the original model. Figure 8(a) is the $1 / 19$ impeller segment finite element model that contains only one blade. Figure $8(\mathrm{~b})$ is the whole finite element model. Assuming that there is no heat exchange with the outside 


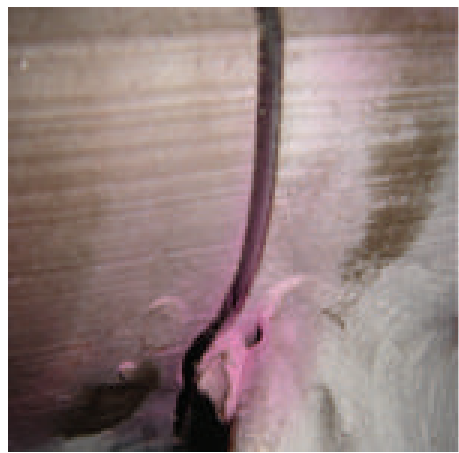

(a) Failure mode 1

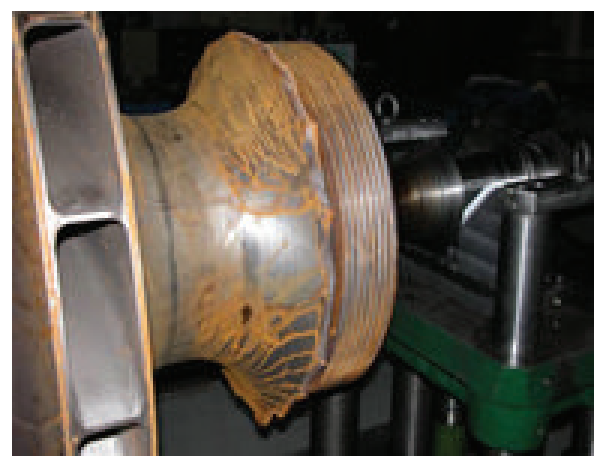

(b) Failure mode 2

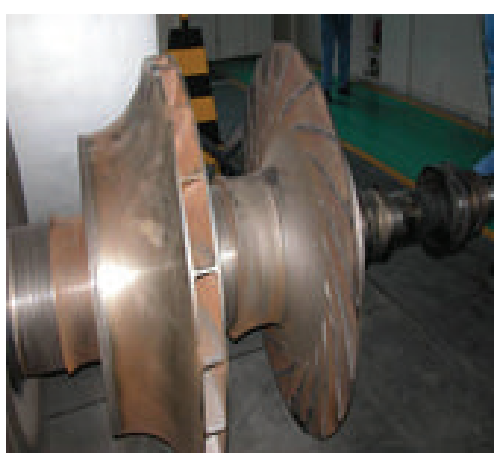

(c) Failure mode 3

Figure 5: Impeller failure modes.

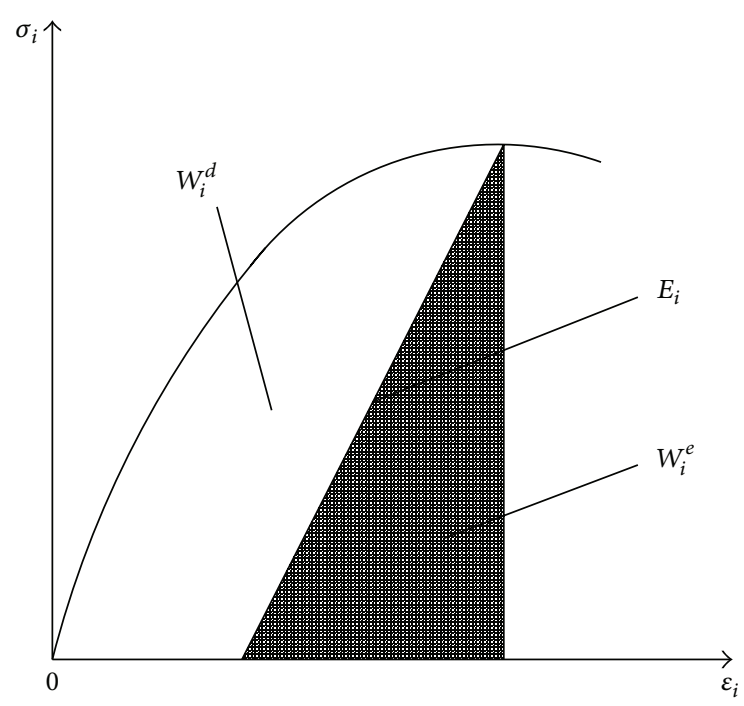

FIGURE 6: The value of the element energy dissipation and releasable strain energy per unit volume.

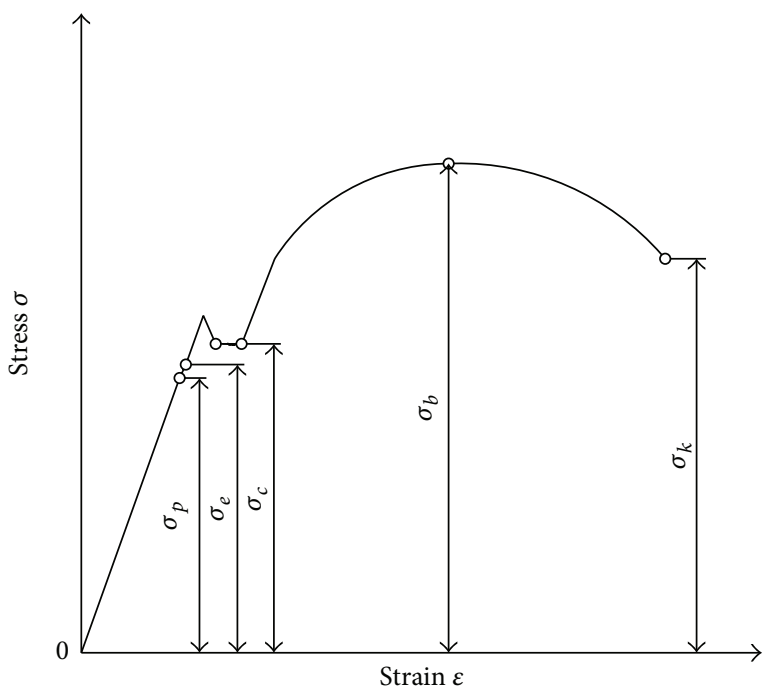

Figure 7: The curve of stress-strain.
TABLE 1: Impeller material properties (density: $7850 \mathrm{~kg} / \mathrm{m}^{3}$ ).

\begin{tabular}{lcccc}
\hline $\begin{array}{l}\text { Temperature } \\
\left({ }^{\circ} \mathrm{C}\right)\end{array}$ & $\begin{array}{c}\text { Elastic } \\
\text { module } \\
\times 10^{5} \mathrm{MPa}\end{array}$ & $\begin{array}{c}\text { Poisson } \\
\text { ratio }\end{array}$ & $\begin{array}{c}\text { Yield } \\
\text { strength } \\
\mathrm{MPa}\end{array}$ & $\begin{array}{c}\text { Expansion } \\
\text { coefficient } \\
\times 10^{-6} /{ }^{\circ} \mathrm{C}\end{array}$ \\
\hline 20 & 2.10 & 0.276 & 850 & 13 \\
\hline
\end{tabular}

of the impeller during operation, only the effect of the centrifugal at working speed of $5130 \mathrm{r} / \mathrm{min}$ and impact of airflow on import and export are considered.

Impeller material parameters are shown in Table 1.

3.3. Finite Element Numerical Simulation. ANSYS provides the function of calculating element volume $V_{e}$ and strain energy $W_{e}$, where

$$
W_{e}=\int_{0}^{V_{e}} \frac{\sigma_{i j} \varepsilon_{i j}}{2} d V ; \quad V_{e}=\int_{0}^{V_{e}} d V .
$$
$W_{e} / V_{e}$.

The average strain energy density of the element is $\bar{W}_{e}=$

Based on the failure criteria of element elastic strain energy density theory in Section 3.1, an extension of the impeller crack is simulated in this paper, and the elements located in the cracks remain in failure state.

"Element life and death technology" in ANSYS is not really adding or deleting unit again, and the stiffness matrix is multiplied by a small factor. Dead element's load will be zero; it is ineffective against the load vector.

We only consider the steady centrifugal stress corresponding to the operating speed. Figure 9 shows the segments von Mises stress distribution during the operating speed of $5130 \mathrm{r} / \mathrm{min}$. The maximum von Mises stress appears in a section near the mouth ring which is point A. Using "element life and death technology" in ANSYS, the largest strain energy density unit is gradually killed, and the crack extension path of the part section is simulated.

Firstly, the finite element model shown in Figure 6 is established based on the solid model and the finite element model has $n$ elements. After loading and calculating, the strain energy $W_{e}$ and volume $V_{e}$ per unit are obtained. Then average strain energy density $\bar{W}_{e}$ of each element is calculated 


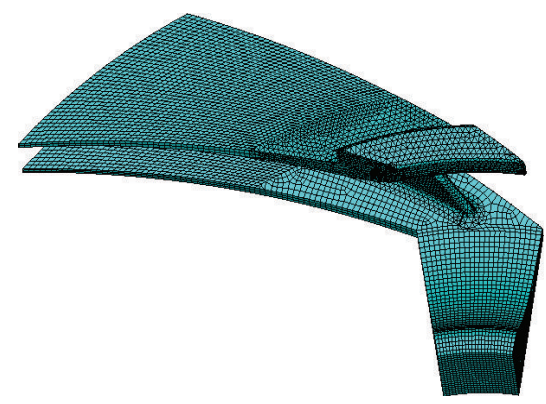

(a) The $1 / 19$ model

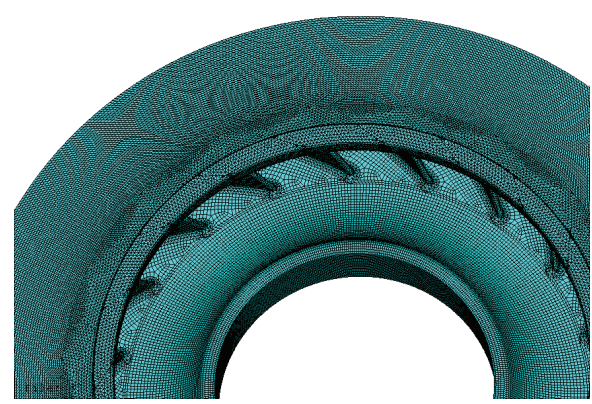

(b) The whole model

Figure 8: Finite element model of the impeller.

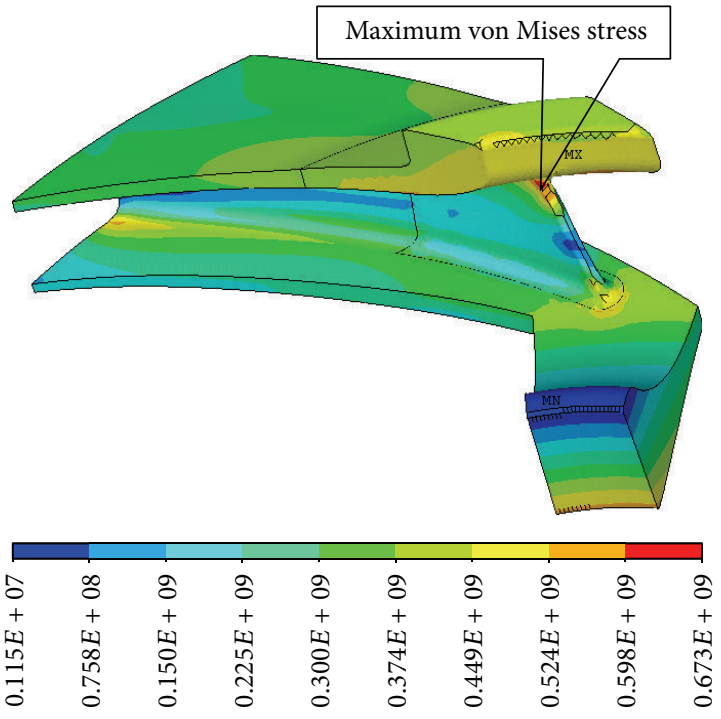

Figure 9: von Mises stress distribution of the impeller.

and arranged finally. The maximum $\bar{W}_{e \max }$ is identified, and the number of the element $n_{e}$ is recorded and killed. Repeating the previous calculating and reloading process, a sequence of unit numbers is obtained. The units killed in the finite element model are reproduced and remarked with the red color. In this way, the crack initiation and extension are simulated. Numerical simulation process of the whole crack extension is shown in Figure 10, and, in order to simulate crack extension perfectly, the finite element model should be finely meshed.

Figure 11 to Figure 15 show the impeller cracking process using the finite element method. The red elements represent the killed elements, and $n m$ in calculation represents the number of units cracked. From the simulation results, Crack initiation point is located on inlet side of shroud and blades weld (point A) (as shown in Figure 11); crack gradually expands along surface to the mouth turn and depth directions until the ring is disconnected completely (as shown in Figure 12). When the cracking unit number $n m$ reaches 2000 , the shroud is totally disconnected (as shown in Figure 13). It is the failure mode 1 .

When the cracks first appear (as shown in Figure 14) at the hub inlet and blade welding joint (point B), cracks

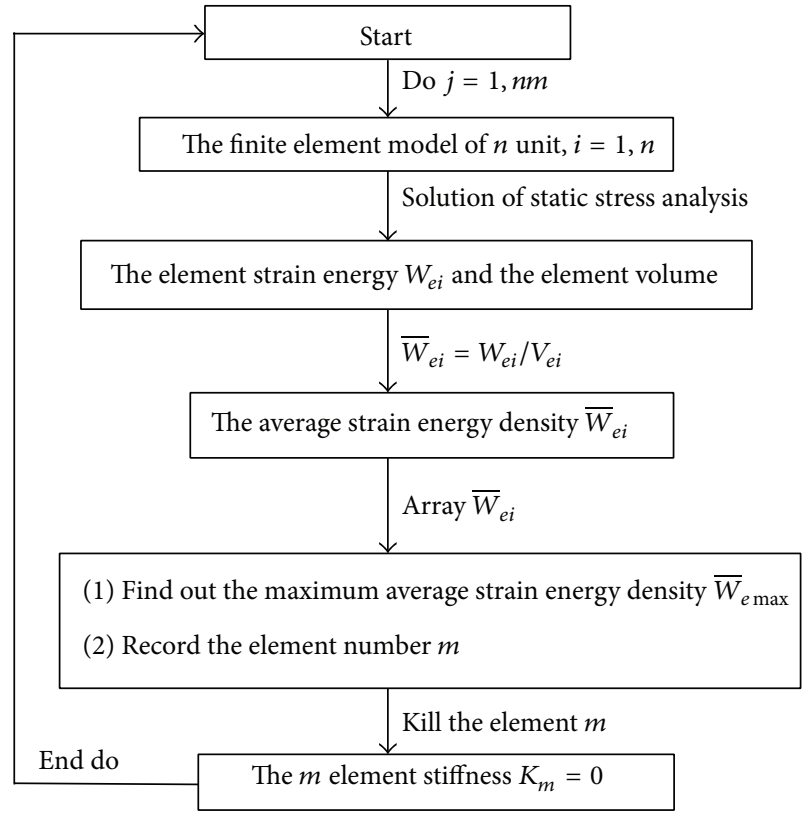

FIGURE 10: The numerical simulation process of crack extension.

extend to both sides along the weld zone, and finally cracks in the circumferential direction adjacent blades are penetrating along cracks extension (as shown in Figure 15). It is the failure mode 2 .

Cracks extension on hub may also have another failure mode. When the weld quality of blades and hub is poor (lack of penetration, such as shown in Figure 16) or there are obvious welding defects near the weld joint of hub inlet and blades, the blade would be gradually torn off inside-out from the disc. It is the failure mode 3.

At the same time, from numerical simulation results, it can be found that the cracks have strong self-similarity, which is consistent with the reality. Therefore it is feasible to adopt the fractal theory to describe crack.

\subsection{Impeller Failure Mechanism Analysis}

3.4.1. Criterion for the Avoiding Rate. The impellers suffer centrifugal force due to high-speed rotation and the airflow of the impact load. Airflow pressure is constantly changing 


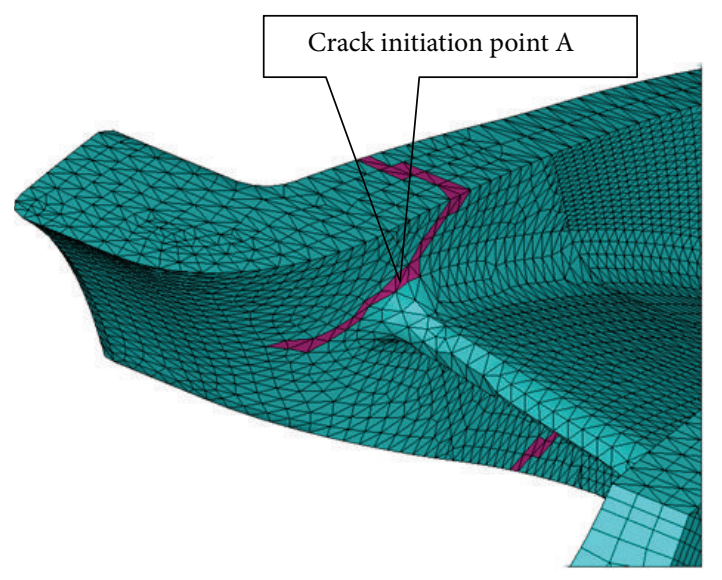

FIGURE 11: Initial crack direction of the impeller $(n m=500)$.

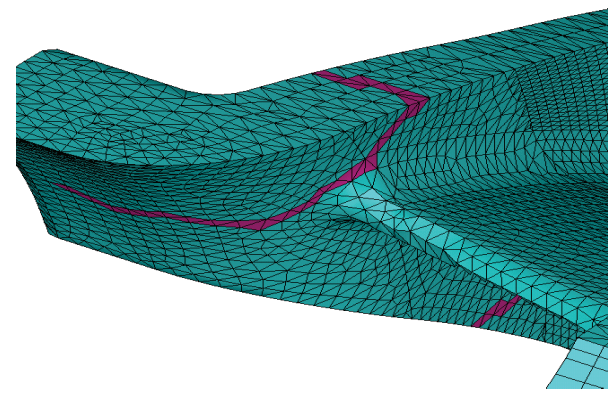

FIGURE 12: Direction of crack extension near the eye of the impeller $(n m=1000)$.

when compressor is operating which is regarded as the dynamic load for the impeller. A large number of studies show that there are two types of working stress that affect fatigue life of the impeller and the blade: steady and alternating stress. Any excessively high stress will result in reduced fatigue life. The steady stress can be reduced through structural optimization design, and the alternating stress is mainly reduced by modulating frequency.

For the excitation frequency $f_{F}$ that needs to meet certain avoiding rate as $x \%$, the adjacent frequency differences $\Delta f_{n}$ and $f_{F}$ need to meet the following relationship:

$$
\begin{gathered}
\Delta f_{n} \geq 2 \times f_{F} \times x \%, \\
f_{n-1}<f_{F}-\frac{\Delta f_{n}}{2}, \quad f_{F}+\frac{\Delta f_{n}}{2}<f_{n} .
\end{gathered}
$$

Equation (10) is the criterion for the avoiding rate between frequency of exciting force and natural frequency. At the same time, it can be obtained that the maximum investigated frequency $f_{n}$ is not less than $f_{F}+\Delta f_{n} / 2$.

3.4.2. Case Study of the Impeller. During the impeller operation, there is always unsteady alternating stress adding to the steady work stress. The steady work stress is mainly due to the centrifugal force of the impeller and has been fully considered during the design. The unsteady alternating stress is mainly caused by random load in actual operation. It can

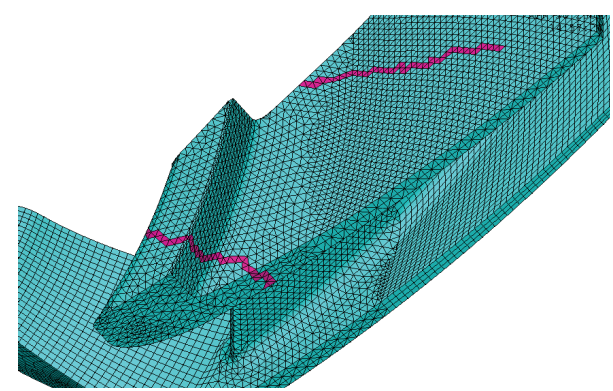

FIGURE 13: The crack that is completely through the eye of the impeller $(n m=1500)$.

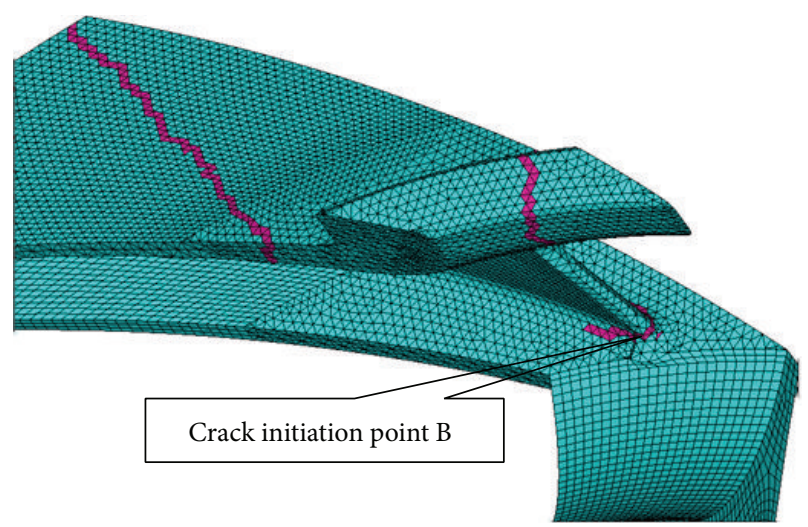

FIGURE 14: The hub crack initiation point and extending direction $(n m=2000)$.

be seen in Figure 9 that the impeller maximum von Mises stress is $673 \mathrm{MPa}$, and yield strength of the impeller material is $850 \mathrm{MPa}$ in Table 1. The impeller von Mises stress at steadystate operation does not reach yield strength of material, and it can be seen that the static strength design is reasonable.

As can be seen from the sectional view of the compressor, there is a backflow structure in front of the third-stage impeller inlet and has 14 blades. The whole impeller has 19 blades. The natural frequency needs to meet at least $8 \%$ avoiding rate with the exciting force frequency. There are two types of airflow exciting force of the impeller as follows. blades:

The exciting force caused by the backflow structure

$$
f_{1}=\frac{5130}{60} \times 14=1197(\mathrm{~Hz}),
$$

$\Delta f_{n 1} \geq 2 \times f_{F} \times x \%=2 \times 1197 \times 8 \%=191.52(\mathrm{~Hz})$.

The exciting force caused by the impeller blades:

$$
f_{2}=\frac{5130}{60} \times 19=1624.5(\mathrm{~Hz}),
$$

$$
\Delta f_{n 2} \geq 2 \times f_{F} \times x \%=2 \times 1624.5 \times 8 \%=259.2(\mathrm{~Hz}) .
$$

From Section 3.4.1, we at least need to consider avoiding rate when the natural frequency of impeller and exciting frequency range $0-1755 \mathrm{~Hz}$. 


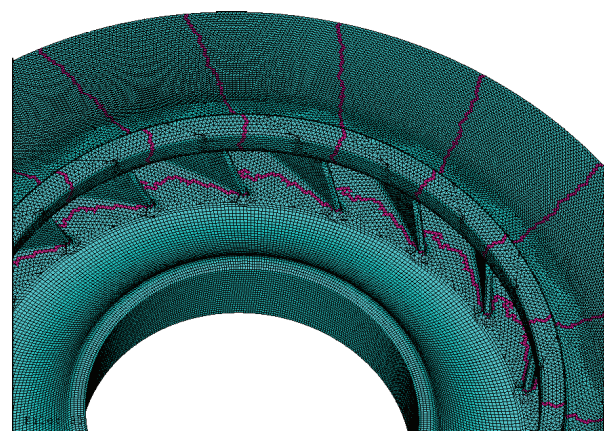

(a) The shroud

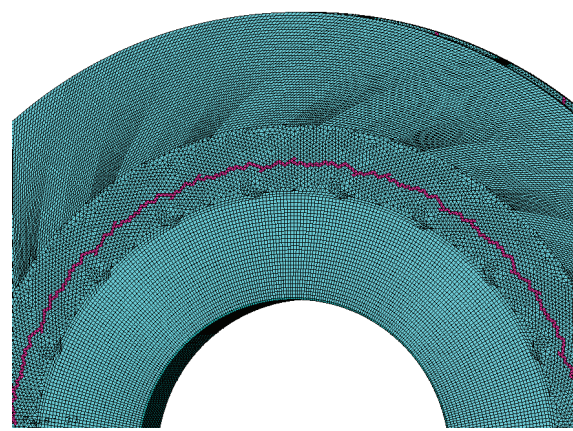

(b) The hub

Figure 15: Impeller final fracture mode $(n m=2500)$.

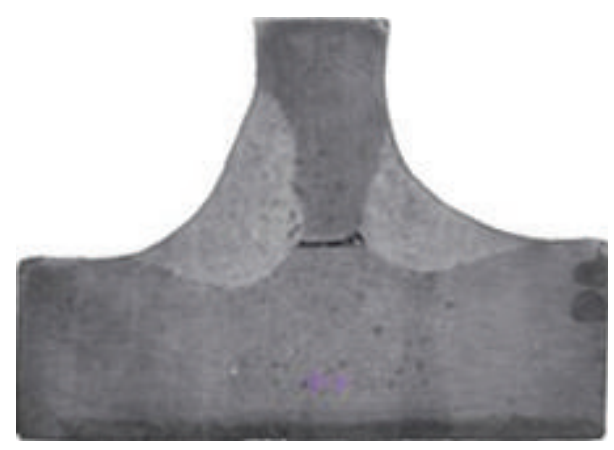

FIGURE 16: Incomplete penetrated corner joints of impeller blade.

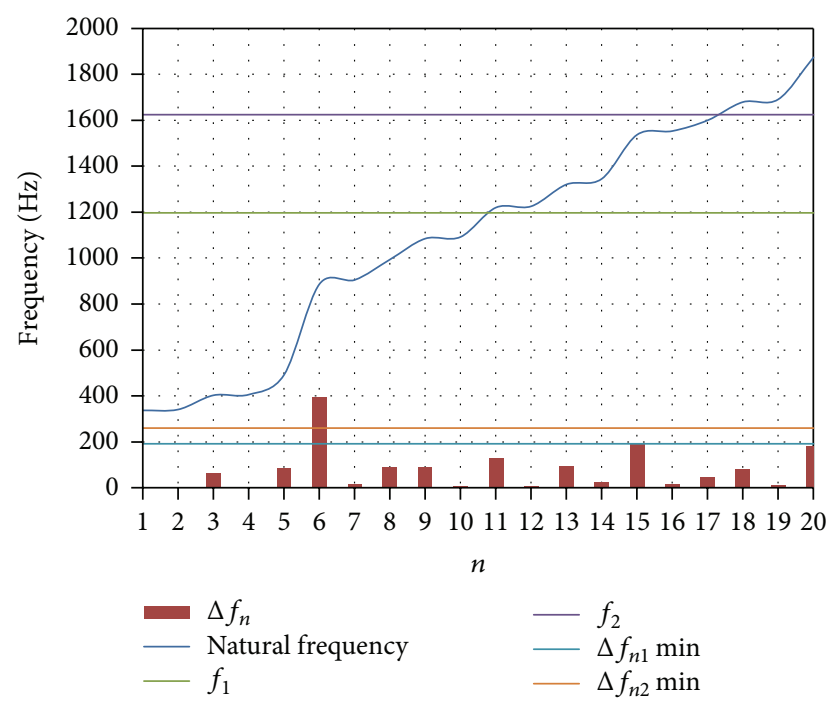

Figure 17: Frequency analysis charts.

The natural frequency value can be obtained by modal analysis. From Figure 17, it can be seen that, for exciting force $1, \Delta f_{n 1} \geq 191.52 \mathrm{~Hz}$ and only $\Delta f_{61}=394.6 \mathrm{~Hz}$ meet the requirements, but $f_{6}=886.5 \mathrm{~Hz}<f_{F 1}+\Delta f_{6} / 2=1394.3 \mathrm{~Hz}$, which does not meet the avoiding guidelines formula (10); for exciting force $2, \Delta f_{n 2} \geq 259.2 \mathrm{~Hz} ; \Delta f_{n}$ does not meet the avoiding guidelines formula (10). Original design natural frequency did not avoid the two types of impeller flow excitation force; it is the main reason of impeller failure.

By investigating the impeller vibration modes, there is a pitch vibration (shown in Figure 18) in the 17th mode with frequency of $1599.8 \mathrm{~Hz}$. Reference [1] shows that umbrella vibration and pitch vibration will occur when impeller rigidity is insufficient only. So, it can be seen that the rigidity of impeller is insufficient. There is a design flaw of impeller.

\section{Conclusions}

The failure process of a third-stage impeller is investigated in this paper. The cracks on different locations were described by the fractal theory. The crack extension is simulated using "element life and death technology", together with the element strain energy density theory. The principle for the avoiding rate between frequency of exciting force and natural frequency is established, and the failure mechanism of the impeller is studied. The main conclusions are given as follows.

(1) The impeller cracks have different sizes but show a very strong self-similarity. The fractal theory can be used to describe the impeller crack, which lays the foundation for the analysis.

(2) By analyzing the strength of the impeller, the maximum von Mises stress is only $673 \mathrm{MPa}$ as shown in Figure 9. It is far away from the yield strength of the impeller material. The design of impeller strength can meet the related requirements. The static stress is not the main cause of impeller failure.

(3) By analyzing vibration characteristics as shown in Figure 17, it can be found that the dynamic characteristics of the impeller are not perfect. The fact that the natural frequency of the impeller does not avoid the frequency of the exciting force effectively would cause vibration risk.

(4) By analyzing the impeller model as shown in Figure 18, the rigidity of impeller structure is insufficient, as the pitch vibration mode appears in the investigated frequency range.

(5) Comparing the impeller macroscopic fracture in Figure 16 with failure mode 3 , it is found that the impeller has welding defects, such as imperfect welding, not penetration. 


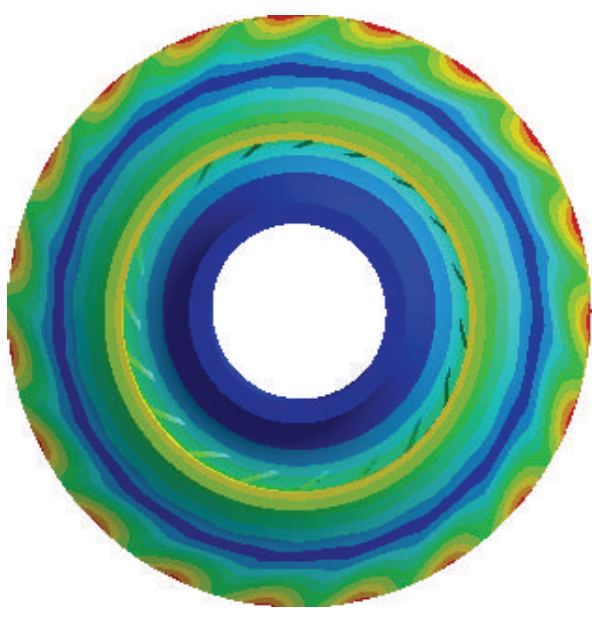

(a) Shroud side

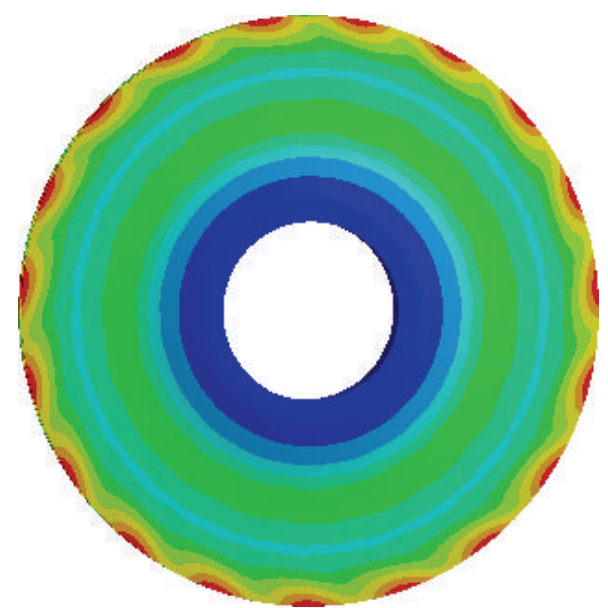

(b) Hub side

FIGURE 18: The 17th mode of impeller.

(6) It can be found that, by using strain energy density, "element life and death technology" together, the crack extension of the impeller can be well established.

\section{Conflict of Interests}

The authors declare that there is no conflict of interests regarding the publication of this paper.

\section{References}

[1] H. Y. Wu, Structural Parts of Turbine and Strength Calculation, Mechanical Industry Press, Beijing, China, 1982.

[2] C. W. Wu, "Reliability analysis of large centrifugal compressor impeller blade fatigue," Equipment and Manufacturing Technology, pp. 1-3, 2008.

[3] N. S. Vyas and J. S. Rao, "Dynamic stress analysis and a fracture mechanics approach to life prediction of turbine blades," Mechanism and Machine Theory, vol. 32, no. 4, pp. 511-527, 1997.

[4] A. Engeda, "The unsteady performance of a centrifugal compressor with different diffusers," Proceedings of the Institution of Mechanical Engineers A: Journal of Power and Energy, vol. 215, no. 5, pp. 585-599, 2001.

[5] N. Ejaz, I. Salam, and A. Tauqir, "Fatigue failure of a centrifugal compressor," Engineering Failure Analysis, vol. 14, no. 7, pp. 13131321, 2007.

[6] T.-G. Kim and H.-C. Lee, "Failure analysis of MVR (machinery vapor recompressor) impeller blade," Engineering Failure Analysis, vol. 10, no. 3, pp. 307-315, 2003.

[7] X.-Y. Yang, Z.-X. Geng, X.-H. Cai, and H.-Q. Shi, "Fracture analysis of the 2nd stage compressor blade in an aeroengine," Journal of Aerospace Power, vol. 16, no. 4, pp. 327-330, 2001.

[8] N. Ejaz, I. Salam, and A. Tauqir, "An air crash due to failure of compressor rotor," Engineering Failure Analysis, vol. 14, no. 5, pp. 831-840, 2007.

[9] L. Wei and Q. Yuan, "Centrifugal compressor surge and control," Fan Technology, vol. 1, pp. 43-47, 2004.

[10] Y. J. Mao, D. T. Qi, and Q. Xu, "Numerical study on high cycle fatigue failure of a centrifugal compressor impeller blades,"
Journal of Xi'an Jiaotong University, vol. 42, no. 11, pp. 1336-1339, 2008.

[11] W. Qin and H. Tsukamoto, "Theoretical study of pressure fluctuations downstream of a diffuser pump impeller-part 1: fundamental analysis on rotor-stator interaction," Journal of Fluids Engineering, Transactions of the ASME, vol. 119, no. 3, pp. 647-652, 1997.

[12] Z.-L. Xu, J.-P. Park, and S.-J. Ryu, "Failure analysis and retrofit design of low pressure 1st stage blades for a steam turbine," Engineering Failure Analysis, vol. 14, no. 4, pp. 694-701, 2007.

[13] C. W. Wu, C. K. Guan, and X. L. Guo, "Large centrifugal compressor impeller blade fatigue reliability analysis," Equipment Manufacturing Technology, vol. 8, no. 1, article 3, 2008.

[14] X. Y. Yang and Z. X. Geng, "Study of a two engine compressor blade failure analysis following the crack," Aerospace, vol. 16, no. 4, pp. 327-330, 2001.

[15] M. E. Ritchie and H. Olff, "Spatial scaling laws yield a synthetic theory of biodiversity," Nature, vol. 400, no. 6744, pp. 557-560, 1999.

[16] L. Lizzi, R. Azaro, G. Oliveri, and A. Massa, "Multiband fractal antenna for wireless communication systems for emergency management," Journal of Electromagnetic Waves and Applications, vol. 26, no. 1, pp. 1-11, 2012.

[17] R. P. Taylor, A. P. Micolich, and D. Jonas, "Fractal analysis of Pollock's drip paintings," Nature, vol. 399, no. 6735, p. 422, 1999.

[18] L. A. Mirny, "The fractal globule as a model of chromatin architecture in the cell," Chromosome Research, vol. 19, no. 1, pp. 37-51, 2011.

[19] S. W. McDonald, C. Grebogi, E. Ott, and J. A. Yorke, "Fractal basin boundaries," Physica D: Nonlinear Phenomena, vol. 17, no. 2, pp. 125-153, 1985.

[20] H.-L. Zou, J.-X. Xu, and J. Jiang, "New bifurcations of basin boundaries involving wada and a smooth wada basin boundary," Chinese Physics B, vol. 17, no. 1, pp. 117-124, 2008.

[21] H.-L. Zou, M. Li, C.-H. Lai, and Y.-C. Lai, "Origin of chaotic transients in excitatory pulse-coupled networks," Physical Review E: Statistical, Nonlinear, and Soft Matter Physics, vol. 86, no. 6, Article ID 066214, 2012. 
[22] F. Erdogan and G. C. Sih, "On crack extension in plane loading and transverse shear," Journal of Basic Engineering, vol. 85, no. 2, pp. 519-527, 1963.

[23] G. C. Sih, "Energy-density concept in fracture mechanics," Engineering Fracture Mechanics, vol. 5, pp. 1037-1040, 1973.

[24] G. C. Sih, "Strain-energy-density factor applied to mixed mode crack problems," International Journal of Fracture, vol. 10, no. 3 , pp. 305-321, 1974.

[25] L. P. Gan, "Based on the total strain energy density roulette low cycle fatigue life and reliability of the model study," Chinese Scientific Papers, vol. 7, no. 8, pp. 616-621, 2012.

[26] Q. Sun, S.-C. Li, X.-D. Feng, W.-T. Li, and C. Yuan, "Study of numerical simulation method of rock fracture based on strain energy density theory," Rock and Soil Mechanics, vol. 32, no. 5, pp. 1575-1582, 2011.

[27] H. P. Xie, "Based on the energy dissipation and the rock strength and overall failure criterion release principle," Rock Mechanics and Engineering, vol. 24, no. 17, pp. 3003-3010, 2005. 


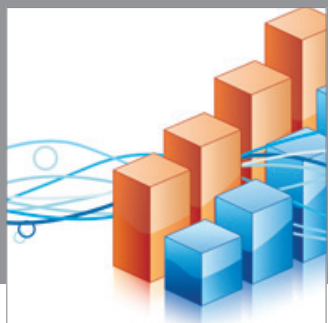

Advances in

Operations Research

mansans

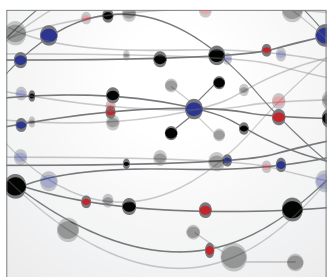

The Scientific World Journal
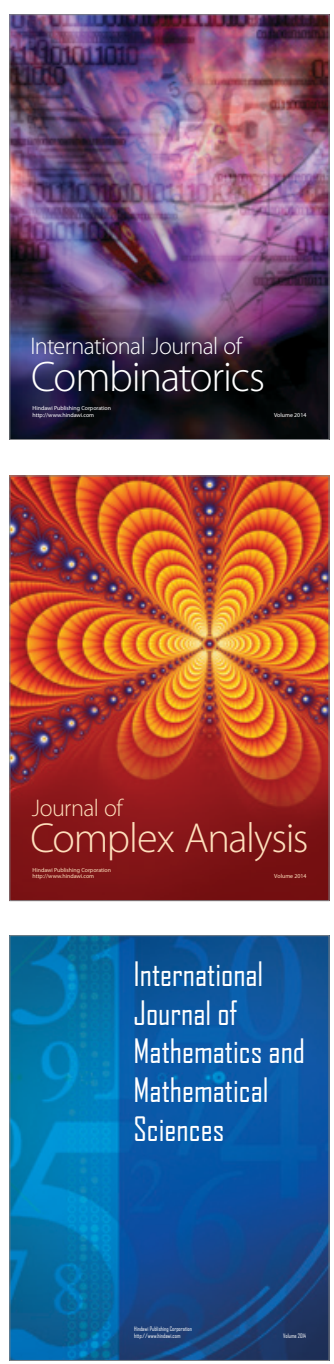
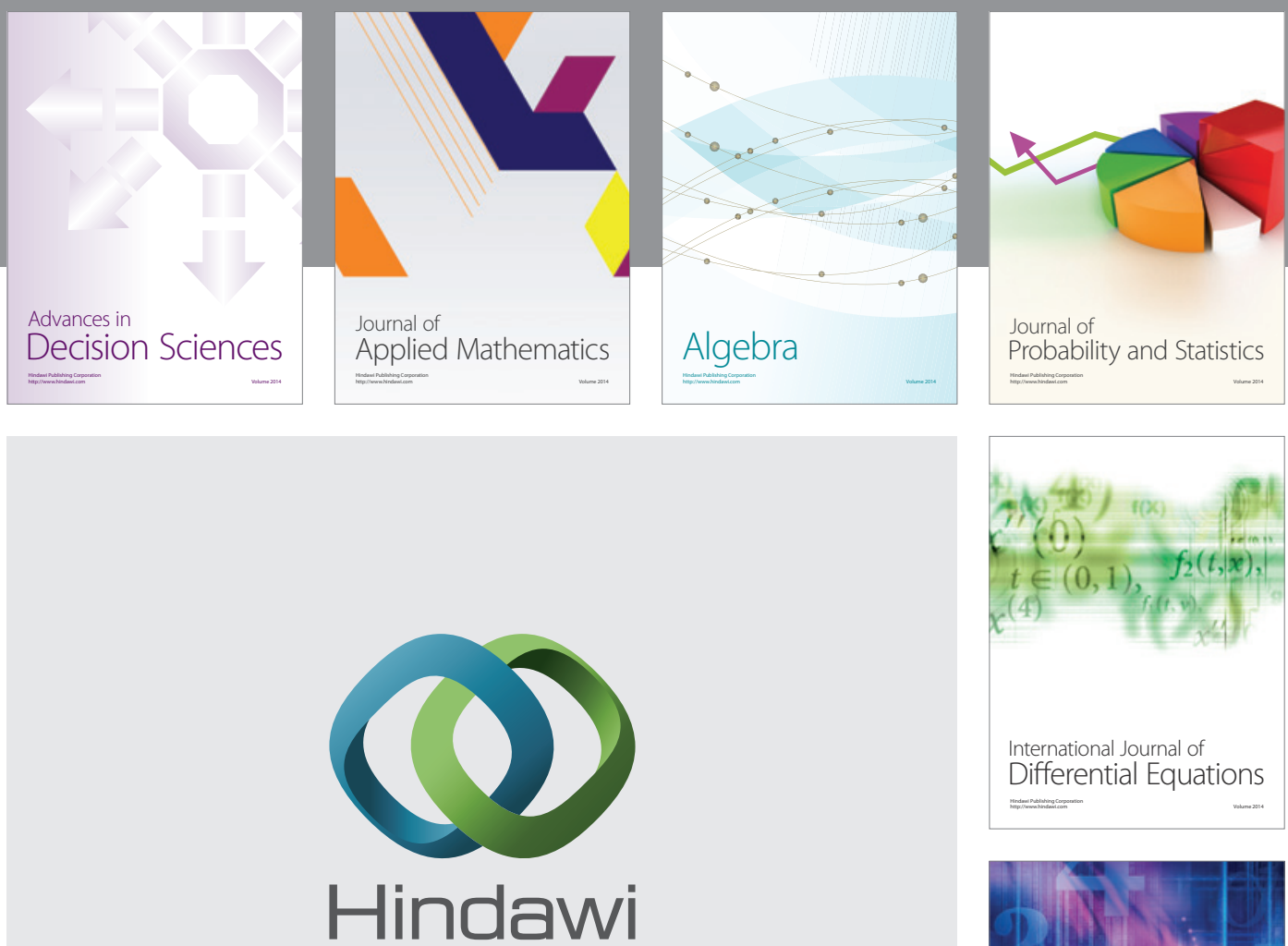

Submit your manuscripts at http://www.hindawi.com
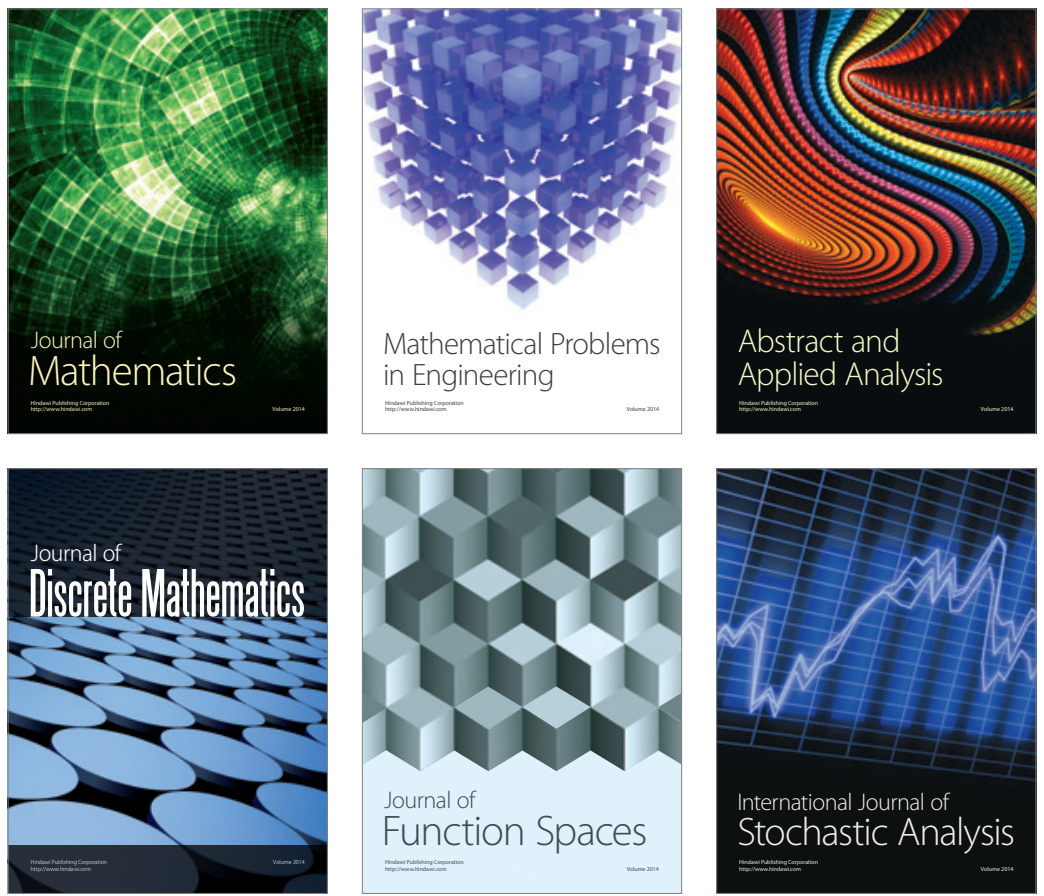

Journal of

Function Spaces

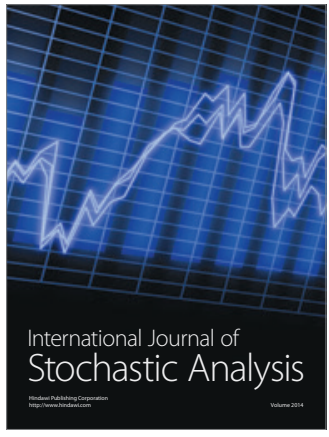

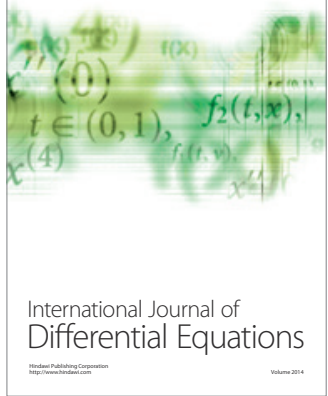
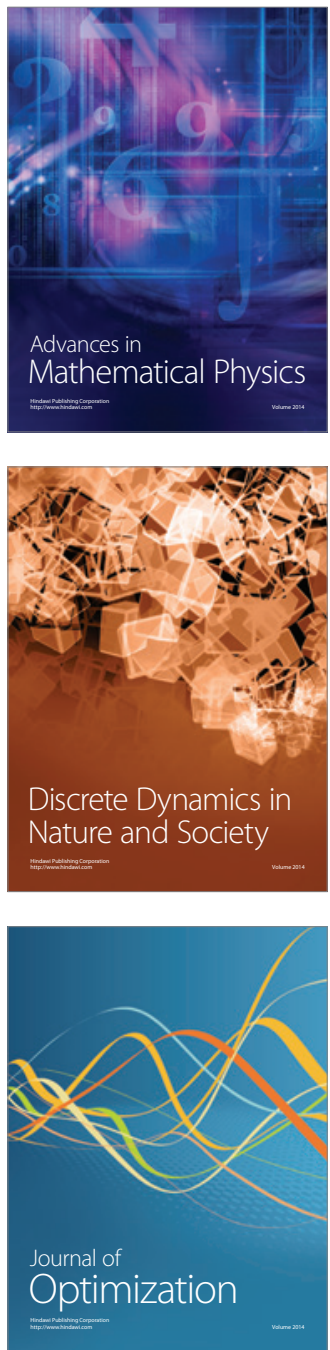